\title{
NEW INEQUALITIES FOR PLANAR CONVEX SETS WITH LATTICE POINT CONSTRAINTS
}

\author{
Poh W. Awyong and Paul R Scott
}

\begin{abstract}
We obtain new inequalities relating the inradius of a planar convex set with interior containing no point of the integral lattice, with the area, perimeter and diameter of the set. By considering a special sublattice of the integral lattice, we also obtain an inequality concerning the inradius and area of a planar convex set with interior containing exactly one point of the integral lattice.
\end{abstract}

\section{INTRODUCTION}

Let $K$ be a compact, planar convex set with interior $K^{\circ}$, and having area $A=$ $A(K)$, perimeter $p=p(K)$, diameter $d=d(K)$ and inradius $r=r(K)$. Let $\Gamma$ denote the integral lattice and let $G\left(K^{o}, \Gamma\right)$ denote the number of points of $\Gamma$ in $K^{o}$. We prove new inequalities relating $A, p, d$ and $r$.

THEOREM 1. Let $K$ be a compact, planar, convex set with $G\left(K^{\circ}, \Gamma\right)=0$. Then

$$
(2 r-1) A \leqslant 2(\sqrt{2}-1) \approx 0.828
$$

with equality when and only when $K$ is congruent to the diagonal square shown in Figure 1.

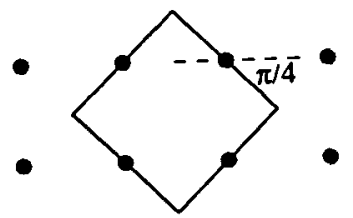

Figure 1.

Received 11th December, 1995

Copyright Clearance Centre, Inc. Serial-fee code: 0004-9729/96 \$A2.00+0.00. 


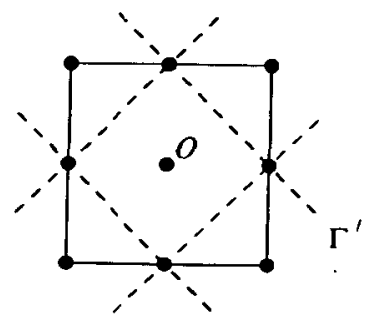

Figure 2.

Corollary 1 . Let $K$ be a compact, planar, convex set with $G\left(K^{o}, \Gamma\right)=1$. Then

$$
(2 r-\sqrt{2}) A \leqslant 4(2-\sqrt{2}) \approx 2.343,
$$

with equality when and only when $K$ is the square shown in Figure 2.

THEOREM 2. Let $K$ be a compact, planar, convex set with $G\left(K^{o}, \Gamma\right)=0$. Then

$$
\begin{gathered}
(2 r-1)|A-1|<\frac{1}{2} \\
(2 r-1)|p-4|<2 \\
(2 r-1)(d-1)<1
\end{gathered}
$$

The limiting infinite strip shows that the stated bounds are best possible.

\section{Proofs of Theorem 1 and Corollary 1}

We first prove two useful lemmas.

Lemma 1. Let $X_{l}$ be the Steiner symmetral of $X$ with respect to the line $l$. Then $r\left(X_{l}\right) \geqslant r(X)$.

Proof: We first show that if $K \subseteq X$, then $K_{l} \subseteq X_{l}$. Let $P Q$ be any chord of $K$ perpendicular to $l$. Since $K \subseteq X$, the line $P Q$ intersects $X$ in a chord $A B$ with $|P Q| \leqslant|A B|$. Now Steiner symmetrisation maps chord $P Q$ to a chord $P^{\prime} Q^{\prime}$ on the line $P Q$, and having its midpoint on $l$ (see for example [1, p.90]). Similarly, the chord $A B$ is mapped to the chord $A^{\prime} B^{\prime}$ on the line $P Q$ and having midpoint on $l$. Since $|P Q| \leqslant|A B|$, the chord $P^{\prime} Q^{\prime}$ is a subset of the chord $A^{\prime} B^{\prime}$. Hence $K_{l} \subseteq X_{l}$.

Now let $C$ be an incircle of $X$. Then $C \subseteq X$ and $C_{l} \subseteq X_{l}$. But $C_{l}$ is congruent to $C$. It follows that $X_{l}$ contains a circle of radius $r(X)$. Therefore $r\left(X_{l}\right) \geqslant r(X)$.

LEMмA 2. Let $K$ be a compact, planar, convex set with $G\left(K^{\circ}, \Gamma\right)=0$. Then there is a compact convex set $K_{*}$ with $G\left(K_{*}^{o}, \Gamma\right)=0$ satisfying the following conditions:

(a) $A\left(K_{*}\right)=A(K), r\left(K_{*}\right) \geqslant r(K)$,

(b) $K_{*}$ is symmetric about the lines $x=1 / 2, y=1 / 2$. 
Proof: We use Steiner symmetrisation to obtain the set $K_{*}$. We first symmetrise $K$ with respect to the line $x=1 / 2$ to obtain the set $K_{1}$. We recall that Steiner symmetrisation preserves convexity and areas so that $K_{1}$ is a convex set with $A\left(K_{1}\right)=$ $A(K)$. Furthermore, by Lemma $1, r\left(K_{1}\right) \geqslant r(K)$.

We now show that $G\left(K_{1}^{\circ}, \Gamma\right)=0$. Since $G\left(K^{\circ}, \Gamma\right)=0, K^{\circ}$ intersects the line $y=k$, where $k$ is an integer, either in the empty set or in a line segment of length at most 1 . Hence the symmetric set $K_{1}^{o}$ intersects the line $y=k$ either in the empty set or between the points $(0, k)$ and $(1, k)$. Clearly, $G\left(K_{1}^{o}, \Gamma\right)=0$.

We now symmetrise $K_{1}$ with respect to the line $y=1 / 2$ to obtain $K_{*}$. Using the same arguments as above, we have $A\left(K_{*}\right)=A\left(K_{1}\right), r\left(K_{*}\right) \geqslant r\left(K_{1}\right)$ and $G\left(K_{*}^{o}, \Gamma\right)=0$. Hence $A\left(K_{*}\right)=A(K)$ and $r\left(K_{*}\right) \geqslant r(K)$. By construction, $K_{*}$ is symmetric about the lines $x=1 / 2$ and $y=1 / 2$ and the lemma is proved.

Let $f(K)=(2 r(K)-1) A(K)$. By Lemma 2 we have $f(K) \leqslant f\left(K_{*}\right)$. It therefore suffices to prove Theorem 1 for sets $K$ which are symmetric about the lines $x=1 / 2$ and $y=1 / 2$.

To fully utilise the symmetry of $K$ about the lines $x=1 / 2$ and $y=1 / 2$, we move the origin to the point $(1 / 2,1 / 2)$. If $r \leqslant 1 / 2,(1)$ is trivially true. Hence we may assume that $r>1 / 2$. Since $K^{0}$ does not contain the points $P_{1}(1 / 2,1 / 2)$, $P_{2}(-1 / 2,1 / 2), P_{3}(-1 / 2,-1 / 2)$ and $P_{4}(1 / 2,-1 / 2)$, it follows by the convexity of $K$ that for each $i=1, \ldots, 4, K$ is bounded by a line $l_{i}$ through the point $P_{i}$, with $l_{1}$ and $l_{3}$ having negative slope and $l_{2}$ and $l_{4}$ having positive slope. Furthermore since $K$ is symmetric about the coordinate axes, $K$ is contained in a rhombus $Q$ determined by the lines $l_{i}, i=1, \ldots, 4$. Since $K \subseteq Q, A(K) \leqslant A(Q)$ and $r(K) \leqslant r(Q)$ we have $f(K) \leqslant f(Q)$. It is therefore sufficient to maximise $f(K)$ over the set of all rhombi, $K=Q$, determined by the lines $l_{i}, i=1, \ldots, 4$ (see Figure 3 ).

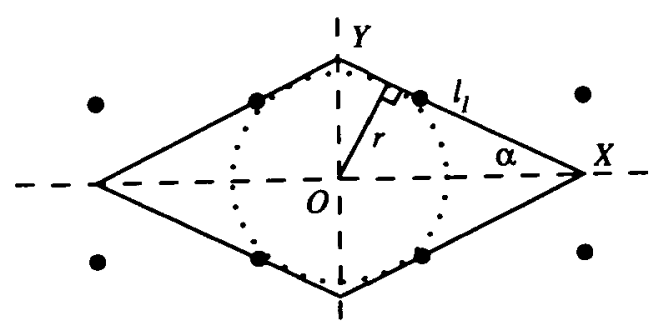

Figure 3.

Let side $l_{1}$ make an acute angle of $\alpha$ with the $x$-axis and let it intercept the $x$ and $y$ axes in the points $X(x, 0)$ and $Y(0, y)$ respectively. Since $l_{1}$ passes through 
$(1 / 2,1 / 2)$, similar triangles give

$$
\frac{y}{x}=\frac{\frac{1}{2}}{x-\frac{1}{2}}
$$

that is,

$$
\frac{1}{x}+\frac{1}{y}=2
$$

Multiplying both sides of the equation by $r$, we get

$$
2 r=\frac{r}{x}+\frac{r}{y}=\sin \alpha+\cos \alpha .
$$

Now

$$
\begin{aligned}
A & =4 . A(\triangle O X Y) \\
& =2 x y \\
& =\frac{2 r^{2}}{\sin \alpha \cos \alpha} \\
& =\frac{4 r^{2}}{(\sin \alpha+\cos \alpha)^{2}-1} \\
& =\frac{4 r^{2}}{4 r^{2}-1} \\
& =1+\frac{1}{4 r^{2}-1}
\end{aligned}
$$

Hence

$$
f(K)=2 r-1+\frac{1}{2 r+1}=g(r)
$$

Now $(1 / 2) g^{\prime}(r)=1-1 /(2 r+1)^{2}>0$. Hence $g$ is an increasing function of $r$. Noting that $1 / 2<r \leqslant \sqrt{2} / 2$, the maximal value of $g$ is therefore attained at $r=\sqrt{2} / 2$, that is, when and only when $K$ is congruent to the diagonal square shown in Figure 1 . In this case

$$
f(K) \leqslant 2(\sqrt{2}-1) \approx 0.828 .
$$

We next use Theorem 1 to prove Corollary 1 . Let $K$ now be a convex set with $G\left(K^{\circ}, \Gamma\right)=1$. Without loss of generality we may assume that the lattice point contained in $K^{\circ}$ is the origin $O$. Let $\Gamma^{\prime}$ be the sublattice of $\Gamma$ with fundamental cell having vertices $(0, \pm 1),( \pm 1,0)$. We first note that $G\left(K^{\circ}, \Gamma^{\prime}\right)=0$ (see Figure 2). Hence letting $A^{\prime}$ and $r^{\prime}$ be the area and the inradius respectively of $K$ measured in the scale of $\Gamma^{\prime}$, and applying (1) to $K$ with respect to $\Gamma^{\prime}$, we have

$$
\left(2 r^{\prime}-1\right) A^{\prime} \leqslant 2(\sqrt{2}-1)
$$


with equality when and only when $K$ is congruent to the square of Figure 2. Since $\Gamma^{\prime}$ is a rotation of $\Gamma$ scaled by a factor of $\sqrt{2}, A^{\prime}=(1 / 2) A$ and $r^{\prime}=(1 / \sqrt{2}) r$ where $A$ and $r$ are the area and the inradius respectively of $K$ measured in the scale of the integral lattice $\Gamma$. Hence

$$
\left(2 \cdot \frac{1}{\sqrt{2}} r-1\right) \frac{A}{2} \leqslant 2(\sqrt{2}-1) .
$$

Simplifying, we get

$$
(2 r-\sqrt{2}) A \leqslant 4(2-\sqrt{2}) \approx 2.343,
$$

with equality when and only when $K$ is congruent to the square of Figure 2 .

\section{Proof of Theorem 2}

We first note that if $r \leqslant 1 / 2$, inequalities (3) and (4) are trivially true. Hence we need only consider those cases for which $1 / 2<r \leqslant \sqrt{2} / 2$.

To prove (3), we first consider $A \leqslant 1$. Since $r>1 / 2$, we have $A>\pi / 4$ and so

$$
(2 r-1)|A-1|=(2 r-1)(1-A)<(\sqrt{2}-1)\left(1-\frac{\pi}{4}\right)<\frac{1}{2} .
$$

Hence we may assume that $A>1$. Using the same arguments as those given in Section 2 , it suffices to consider a set $K$ where $K$ is a rhombus of the type described in Figure 3. Let $Q(r)$ denote such a rhombus with inradius $r$. From (6) we have

$$
(2 r-1)|A-1|=(2 r-1)(A-1)=\frac{1}{2 r+1}<\frac{1}{2} .
$$

Taking the infinite strip to be the limit of $Q(r)$ as $r$ tends to $1 / 2$, it is seen that the stated bound is best possible.

To prove (4), we first consider $p \leqslant 4$. Since $r>1 / 2$, we have $p>\pi$ and so

$$
(2 r-1)|p-4|=(2 r-1)(4-p)<(\sqrt{2}-1)(4-\pi)<2 .
$$

Hence we may assume that $p>4$. We note further that if $K$ is a convex polygon, $K$ may be partitioned into triangles by joining each vertex of $K$ to an in-centre of $K$. Summing the areas of these triangles gives

$$
A \geqslant \frac{1}{2} p r,
$$

with equality when and only when every edge of $K$ touches the unique incircle. Since any compact convex set may be approximated by a convex polygon, this inequality is 
valid for all compact convex sets in the plane. By combining inequality (7) with (3) and noting that $r>1 / 2$, we have

$$
(2 r-1)|p-4|=(2 r-1)(p-4) \leqslant(2 r-1)\left(\frac{2 A}{r}-4\right) \leqslant 4(2 r-1)(A-1) \leqslant 4 . \frac{1}{2}=2,
$$

obtaining (4). As before, taking the infinite strip to be the limit of $Q(r)$ as $r$ tends to $1 / 2$, the stated bound is best possible.

Finally, to prove (5), we note that $(w-1)(d-1) \leqslant 1$ with equality when and only when $K$ is a triangle of the type shown in Figure 4 (see [2]).

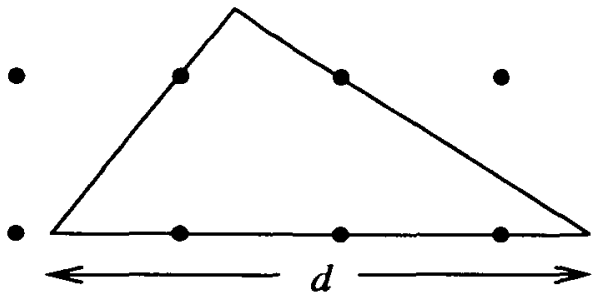

Figure 4.

Since $w \geqslant 2 r$, we have

$$
(2 r-1)(d-1) \leqslant(w-1)(d-1) \leqslant 1 .
$$

Taking the infinite strip to be the limit of a sequence of triangles of the type shown in Figure 4 as $w$ tends to $2 r$, it can be seen that the stated bound is best possible.

\section{REFERENCES}

[1] H.G. Eggleston, Convexity (Cambridge University Press, Cambridge, 1958).

[2] P.R. Scott, 'Two inequalities for convex sets with lattice point constraints in the plane', Bull. London Math. Soc. 11 (1979), 273-278.

[3] P.R. Scott, 'Further inequalities for convex sets with lattice point constraints', Bull. Austral. Math. Soc. 21 (1980), 7-12.

\footnotetext{
Department of Pure Mathematics

The University of Adelaide

South Australia 5005

e-mail: pawyong@maths.adelaide.edu.au pscott@maths.adelaide.edua.au
} 